\title{
Solving the phase problem in solution scattering
}

\author{
Thomas D. Grant ${ }^{1,2}$ \\ ${ }^{1}$ SUNY University at Buffalo, Buffalo, NY \\ ${ }^{2}$ Hauptman-Woodward Institute, Buffalo, NY
}

Small angle scattering is an experimental technique used to analyze the molecular structures of a wide variety of biological and non-biological samples in solution. In contrast to X-ray crystallography and cryoelectron microscopy, where 3D electron density maps are calculated, available methods for generating 3D structural information from 1D solution scattering data rely exclusively on modeling. Many modeling algorithms rely on an implicit assumption that electron density is uniform inside the particle envelope. This assumption breaks down at resolutions better than approximately $10-15 \AA$ where fluctuations in electron density contribute significantly to scattering and for particles with large scale conformational dynamics or containing mixed density species. Here I present a method ${ }^{1}$ for calculating electron density maps directly from solution scattering data. Using only few simple restraints such as solvent flattening, this method avoids many of the assumptions limiting the resolution and accuracy of conventional modeling algorithms. The algorithm has been applied to publicly available experimental scattering data from twelve different biological macromolecules. In each case the electron density maps closely match known atomic models, including complex shapes with multiple density components. These results demonstrate that accurate and complex electron density maps can be reconstructed from small angle scattering data and with significantly fewer restraints than imposed by existing modeling methods.

${ }^{1}$ Grant, T. D. (2018). Ab initio electron density determination directly from solution scattering data. Nature Methods 15, 191-193.

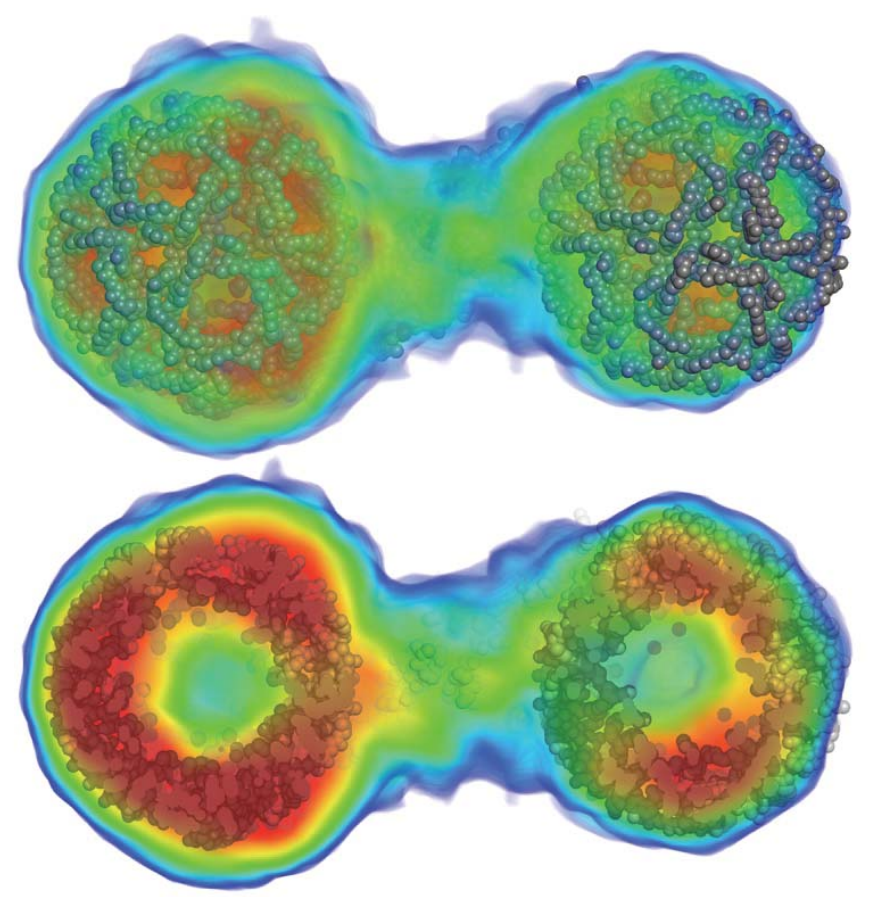

